\title{
A novel LARGE1-AFF2 fusion expanding the molecular alterations associated with the methylation class of neuroepithelial tumors with PATZ1 fusions
}

\author{
Arnault Tauziède-Espariat ${ }^{1,2^{*}}$, Guillaume Chotard ${ }^{3 \dagger}$, François le Loarer ${ }^{4,5,6 \dagger}$, Jessica Baud ${ }^{5 \dagger}$, Rihab Azmani ${ }^{5 \dagger}$, \\ Volodia Dangouloff-Ros ${ }^{7}$, Nathalie Boddaert ${ }^{7}$, Céline Icher-de-Bouyn ${ }^{8}$, Edouard Gimbert ${ }^{9}$, Lauren Hasty ${ }^{1}$, \\ Alice Métais ${ }^{1}$, Fabrice Chrétien ${ }^{1}$ and Pascale Varlet ${ }^{1,2}$ on behalf of on behalf of the the RENOCLIP-LOC
}

\begin{abstract}
A novel DNA methylation class of tumor within the central nervous system, the "neuroepithelial tumor (NET), PATZ1 fusion-positive" has recently been identified in the literature, characterized by EWSR1- and MN1-PATZ1 fusions. The cellular origin of this tumor type remains unknown, wavering between glioneuronal or mesenchymal (as round cell sarcomas with EWSR1-PATZ1 of the soft tissue). Because of the low number of reported cases, this tumor type will not be added to the 2021 World Health Organization Classification of Tumors of the Central Nervous System (CNS). Herein, we report one case of a CNS tumor classified by DNA methylation analysis as NET-PATZ1 but harboring a novel LARGE1-AFF2 fusion which has until now never been described in soft tissue or the CNS. We compare its clinical, histopathological, immunophenotypical, and genetic features with those previously described in NET-PATZ1. Interestingly, the current case presented histopathological (astroblastoma-like features, glioneuronal phenotype), clinical (with a favorable course), genetic (1 p loss), and epigenetic (DNA-methylation profiling) similarities to previously reported cases of NET-PATZ1. Our results added data suggesting that different histomolecular tumor subtypes seem to be included within the methylation class "NET, PATZ1 fusion-positive", including non PATZ1 fusions, and that further cases are needed to better characterize them.
\end{abstract}

Keywords: LARGE1, AFF2, PATZ1, Neuroepithelial tumor

\section{Introduction}

Neuroepithelial tumors (NET) with PATZ1 fusions (NETPATZ1) have been isolated by a distinct DNA methylation profile and are characterized by recurrent fusions of PATZ1 in association with EWSR1 or MN1 genes [1].

\footnotetext{
*Correspondence: a.tauziede-espariat@ghu-paris.fr

${ }^{\dagger}$ Guillaume Chotard, François le Loarer, Jessica Baud and Rihab Azmani

have contributed equally to this work

${ }^{1}$ Department of Neuropathology, GHU Paris - Psychiatry

and Neuroscience, Sainte-Anne Hospital, 1, rue Cabanis, 75014 Paris, France

Full list of author information is available at the end of the article
}

These tumors present a wide variety of morphologies and immunophenotypes, having been initially classified as glioneuronal tumors, astroblastomas, ependymomas, glioblastomas, pleomorphic xanthoastrocytomas, primary neuroepithelial tumors, and round cell sarcomas, with different histopathological grades [2-11]. Because extra-CNS sarcomas may also harbor an EWSR1-PATZ1 fusion and because of the uncertainty of the cellular origin of NET-PATZ1, this tumor type will not be added to the upcoming edition of the World Health Organization Classification of CNS Tumors [12]. Here we report a temporal tumor with a NET-PATZ1 DNA methylation class 
(MC) but harboring a LARGE1-AFF2 fusion. We compare its clinical, histopathological, immunophenotypical, genetic and epigenetic features with those previously described in NET-PATZ1.

\section{Case presentation}

A 3-year-old girl began experiencing seizures. Cerebral magnetic resonance imaging (MRI) showed a left temporal mass with a hyperintense signal on T1-weighted images, a hypointense signal on T2-weighted-images and with a homogeneous and intense contrast enhancement after gadolinium injection (Fig. 1A-D). The mass was cortical, well-circumscribed, solid with a small cyst, having slight perilesional edema and no leptomeningeal attachment. Neither hemorrhagic nor necrotic modification was observed and no calcifications were present on computerized tomodensitometry. Diffusion was not restricted (Fig. 1E). Gross total resection was achieved. Microscopically, the tumor was well-delineated from the brain parenchyma (Fig. 1F), and heterogeneous, presenting a sclerous stroma including isolated cells and nodules of glial cells with microcystic changes (Fig. 1G-I). No ependymal nor astroblastic pseudorosettes, or rhabdoid component were evidenced. The cytoplasm of the

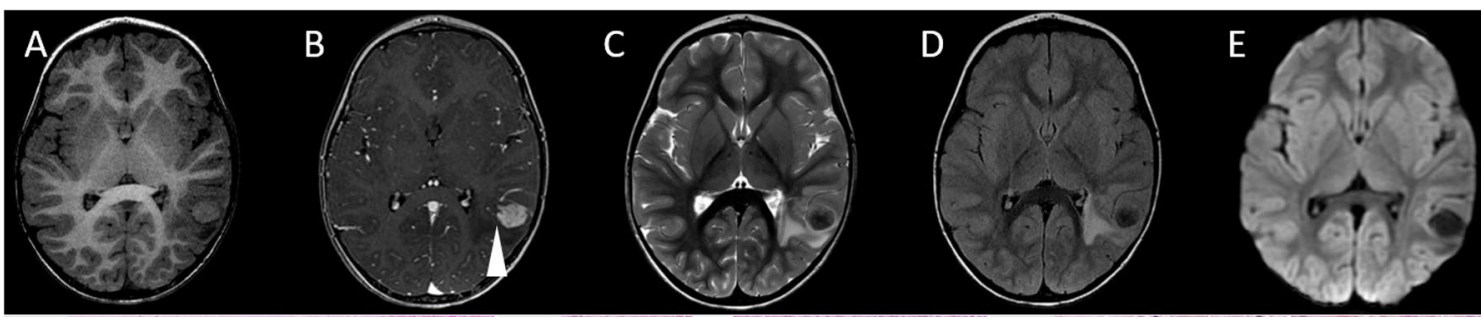

$\mathrm{F}$

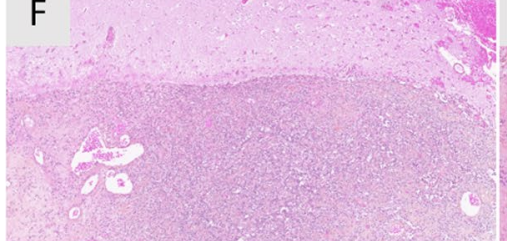

I
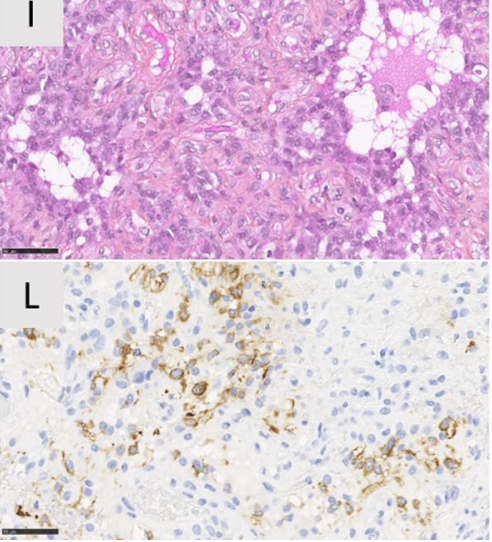

G
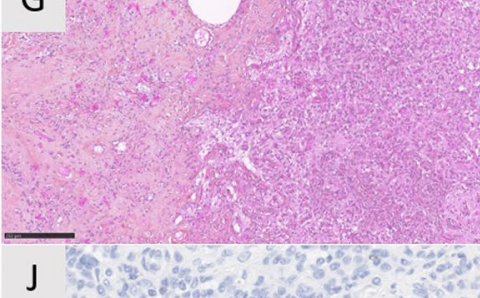

J

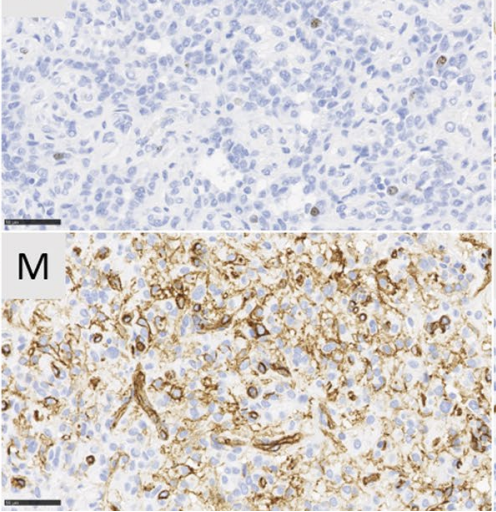

$\mathrm{H}$
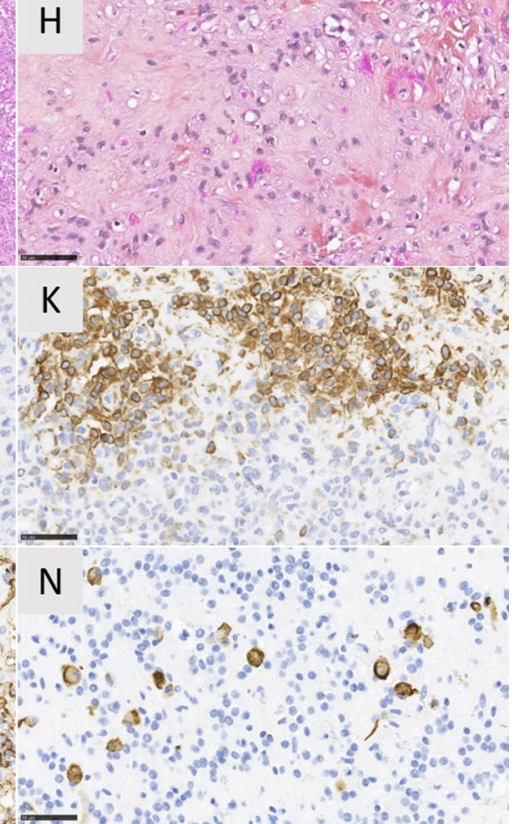

Fig. 1 Radiological and histopathological features. A Axial T1-weighted magnetic resonance imaging image showing a left hyperintense temporal lesion. B Axial T1-weighted magnetic resonance imaging image after contrast injection showing an intense homogeneous enhancement of the lesion which is mainly solid with a small cyst (arrow). C Axial T2-weighted magnetic resonance imaging image showing an hypointensity of the lesion and a perilesional edema. D T2-FLAIR-weighted image showing the vasogenic perilesional edema. E Diffusion was not restricted. F The well delimitation of the tumor (HPS, magnification $\times 50$ ). G The alternance of fibrous stroma containing few tumor cells and highest cellular areas (HPS, magnification $\times 200$ ). $\mathbf{H}$ The collagenous stroma with few spindle cells (HPS, magnification $\times 400)$. I The cellular areas monomorphous cells with round to oval nuclei and abundant eosinophilic cytoplasm with microcystic changes (HPS, magnification $\times 400$ ). J Low MIB-1 labeling index (magnification $\times 400$ ). K GFAP immunoexpression by many tumor cells (magnification $\times 400$ ). L MAP2 immunoexpression by a subset of tumor cells (magnification $\times 400$ ). $\mathbf{M}$ Diffuse extravascular CD34 immunoexpression with CD34-positive ramified processes (magnification $\times 400)$. $\mathbf{N}$ Desmin immunoexpression by some tumor cells (magnification $\times 400$ ). Black scale bars represent $500 \mu \mathrm{m}$ for figure F, $250 \mu \mathrm{m}$ for figure $\mathrm{G}$, and $50 \mu \mathrm{m}$ for figure H-N. HPS: Hematoxylin Phloxin Saffron. 
tumor cells was abundant and eosinophilic. Some tumor cells were plurinucleated. No mitotic figures, nor necrosis or microvascular proliferation were observed and the MIB-1 labeling index was low (around 5\%) (Fig. 1J). Very few perivascular inflammatory infiltrates were present but no eosinophilic granular bodies or ganglion cells were observed. The tumor cells expressed glial markers (GFAP and Olig2 for a subset of cells; Fig. 1K), MAP2 (Fig. 1L) and NeuN (without expression of synaptophysin and chromogranin A), and CD34 (Fig. 1M). There was no immunopositivity for CKAE1/AE3, CK18, CD99, BCOR, SOX10, IDH1R132H or Lin28A. The tumor cells focally expressed desmin but there was no immunoreactivity for smooth muscle actin or myogenin (Fig. 1N). The expression of ATRX, BRG1, INI1 and H3K27me3 was retained. Because of the fibrous stroma, a diagnosis of astroblastoma was initially suggested but a FISH analysis of the $M N 1$ gene failed to reveal a rearrangement. RNA sequencing (including EWSR1, MN1 and PATZ1 genes) evidenced a $L A R G E 1-A F F 2$ gene fusion (Fig. 2A) and a DNA methylation analysis was conducted. The tumor was classified as NET-PATZ1 (with a strong calibrated score of 0.99) based on the DNA methylation profiling using a random forest machine learning classification algorithm as previously described (v12.3; Fig. 2B) [13]. Twelve months later, the patient is well without adjuvant treatment and no residual tumor on MRI.

\section{Discussion and conclusions}

Here, we report an intracerebral tumor harboring a novel LARGE1-AFF2 fusion, with clinical, radiological, histopathological, and epigenetic similarities to NET-PATZ1. Like most NET-PATZ1, our observation concerned a supratentorial tumor in a child (Table S1) $[1-8,11]$. Whereas neuroradiological data of this recently described tumor type is scarce, our case presented as a solid and cystic lesion with T2-weighted hypointensity suggesting fibrotic content, well-circumscribed from the brain parenchyma, as previously reported $[4,8,11]$. NET-PATZ1 encompassed a wide variety of morphologies in the literature, including glial, glioneuronal, embryonal tumors and sarcomas (Table S1). Based on the literature review and our case, the presence of a collagenous stroma and microcysts seem to be frequent in NET-PATZ1 (Table S1) [1, 4, 8, 11]. Because of this pattern and because some of them present pseudorosettes (not seen in our case), pathologists tend to consider them a differential diagnosis for astroblastoma, MN1-fused [1, 2, 8]. However, NET$P A T Z 1$, as with our case, exhibit frequent glioneuronal immunoprofiles and an extravascular expression of
CD34 may be found, which is rare in astroblastomas (Table S1) [1, 11, 14]. The histopathological and epigenetic distinction between sarcomas with EWSR1PATZ1 fusion and NET, PATZ1-fusion positive is still not clear. Indeed, CNS and extra-CNS tumors with PATZ1 fusion share some histopathological features (microcysts, collagenous stroma and pseudorosettes, and a mesenchymal component described in a part of NET-PATZ1) and a polyphenotypic immunoprofile (expression of glial, neuronal and CD34 in both) [1, 11, 15-17]. The DNA methylation analysis (v12.3) classified our case as a NET, PATZ1, although the tumor did not harbor a PATZ1 fusion. Similarly, the case clustered with $P A T Z 1$-fused sarcomas, located outside the CNS on the whole RNA sequencing analysis. Our case presented a LARGE1-AFF2 fusion which has not been previously reported in CNS or in soft tissue tumors. Whereas no LARGE1 fusion has been described in tumors, several tumor types have been reported with $A F F 2$ fusions in association with different partners (DEK in squamous carcinomas, $R E T$ in lung cancer, STAG2 in T-cell lymphoma) [18-21]. The fusion may drive the oncogenesis by deregulation of transcription as $A F F 2$ encodes a RNA-binding protein through the $\mathrm{C}$-terminal domain that can activate transcription [22]. Moreover, the chimeric protein is predicted to contain the major functional domains of both LARGE1 and AFF2 proteins. Interestingly, LARGE1 (22q12.3) and PATZ1 (22q12.2) genes are found in close proximity on chromosome 22, and PATZ1 was highly expressed at the RNA level (whereas $L A R G E 1$ and $A F F 2$ are not) as observed in sarcomas with PATZ1 fusion. Because DNA methylation profiles are thought to represent a combination of both somatically acquired DNA methylation changes and a signature reflecting the cell of origin [23], it is reasonable to assume that our case represents a subtype of NET-PATZ1. Because of the limited follow-up data and the heterogeneity of treatments applied in NET-PATZ1 cases from the literature, no precise prognosis has been defined [1]. And despite histopathological signs of aggressivity, a probable intermediate grade has been suggested considering the better outcome associated with NET-PATZ1 compared to high-grade tumors [1]. Our case is in line with these data, showing no recurrence one year after gross total resection without adjuvant treatment.

In conclusion, we expanded the defined MC NETPATZ1 genetic spectrum with one novel fusion that does not involve the PATZ1 gene. This case illustrates that further studies are needed to characterize in detail this rare type of tumor in terms of cellular origin, histopathology, genetic features and outcome. 

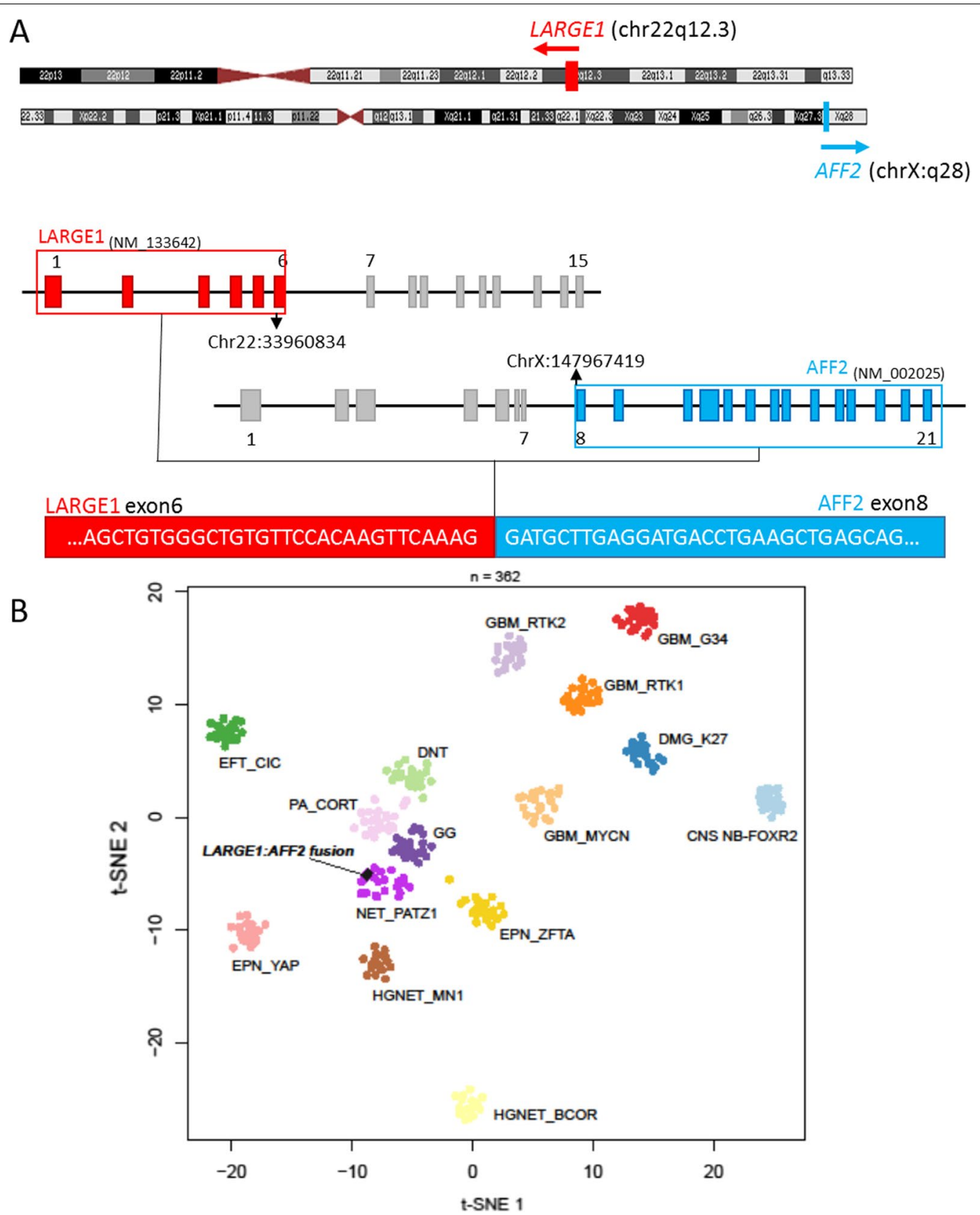

Fig. 2 Illustration of the fusion and t-SNE analysis A RNAseq analysis highlights a fusion between LARGE1 (red) and AFF2 (blue) genes, respectively located on chr22q12.3 and chrX.q28 with a breakpoint in exon 6 for $L A R G E 1$ and exon 8 for AFF2. B t-distributed stochastic neighbor embedding (t-SNE) analysis of the DNA methylation profile of the investigated tumor alongside 361 selected reference samples

\section{Supplementary Information}

The online version contains supplementary material available at https://doi. org/10.1186/s40478-022-01317-8.

Additional file 1. Table S1. Summary of clinical, histopathological and molecular data of CNS PATZ1-fused tumors reported in the literature

\section{Acknowledgements}

We would like to thank the laboratory technicians at the GHU Paris Neuro Sainte-Anne for their assistance, as well as the Integragen platform for their technical assistance with DNA-methylation analyses. We thank P. Sievers from the Department of Neuropathology of the University Hospital Heidelberg for performing an unsupervised t-SNE analysis of the DNA methylation data together with a reference cohort. 


\section{Authors' contributions}

ATE, GC, VDR, NB, Cl, and EG compiled the MRI and clinical records; ATE, GC, $A M, F C$ and PV conducted the neuropathological examinations; FLL, RA and JB conducted the molecular studies; ATE, LH, and PV drafted the manuscript. All authors reviewed the manuscript.

\section{Funding}

The authors declare that they have not received any funding.

\section{Declarations}

\section{Ethics approval and consent to participate}

This study was approved by the GHU Paris Psychiatrie Neurosciences, SainteAnne Hospital's local ethic committee.

\section{Consent for publication}

The patient signed informed consent forms before treatment was started.

\section{Competing interests}

The authors declare that they have no conflicts of interest directly related to the topic of this article.

\section{Author details}

1 Department of Neuropathology, GHU Paris - Psychiatry and Neuroscience, Sainte-Anne Hospital, 1, rue Cabanis, 75014 Paris, France. ${ }^{2}$ Institute of Psychiatry and Neurosciences of Paris (IPNP), Université de Paris, INSERM, U1266, 75014 Paris, France. ${ }^{3}$ Department of Pathology, Groupe Hospitalier Pellegrin, CHU de Bordeaux, Bordeaux, France. ${ }^{4}$ Université de Bordeaux, Talence, France. ${ }^{5}$ Department of Biopathology, Institut Bergonié, Bordeaux, France. ${ }^{6}$ INSERM U1218, ACTION, Institut Bergonié, Bordeaux, France. ${ }^{7}$ Department of Pediatric Radiology, Hôpital Necker Enfants Malades, AP-HP, Université de Paris, INSERM U1163, Institut Imagine, Paris, France. ${ }^{8}$ Department of Pediatric Oncology, Bordeaux University Hospital, Bordeaux, France. ${ }^{9}$ Department of Pediatric Neurosurgery, Bordeaux University Hospital, Bordeaux, France.

Received: 2 December 2021 Accepted: 21 January 2022

Published online: 03 February 2022

\section{References}

1. Alhalabi KT, Stichel D, Sievers P, Peterziel H, Sommerkamp AC, Sturm D et al (2021) PATZ1 fusions define a novel molecularly distinct neuroepithelial tumor entity with a broad histological spectrum. Acta Neuropathol (Berl) 142:841-857

2. Zschernack V, Jünger ST, Mynarek M, Rutkowski S, Garre ML, Ebinger M et al (2021) Supratentorial ependymoma in childhood: more than just RELA or YAP. Acta Neuropathol (Berl) 141:455-466

3. Siegfried A, Rousseau A, Maurage C-A, Pericart S, Nicaise Y, Escudie F et al (2019) EWSR1-PATZ1 gene fusion may define a new glioneuronal tumor entity. Brain Pathol Zurich Switz 29:53-62

4. Rossi S, Barresi S, Giovannoni I, Alesi V, Ciolfi A, Colafati GS, et al. (2020) Expanding the spectrum of EWSR1-PATZ1 rearranged CNS tumors: an infantile case with leptomeningeal dissemination. Brain Pathol Zurich Switz., e12934.

5. Bridge JA, Sumegi J, Druta M, Bui MM, Henderson-Jackson E, Linos K, et al. (2019) Clinical, pathological, and genomic features of EWSR1-PATZ1 fusion sarcoma. Mod Pathol Off J U S Can Acad Pathol Inc., 32:1593-604

6. Pei J, Zhao X, Patchefsky AS, Flieder DB, Talarchek JN, Testa JR, et al. (2019) Clinical application of RNA sequencing in sarcoma diagnosis: an institutional experience. Med (Baltimore), 98:e16031

7. Lopez-Nunez O, Cafferata B, Santi M, Ranganathan S, Pearce TM, Kulich SM et al (2021) The spectrum of rare central nervous system (CNS) tumors with EWSR1-non-ETS fusions: experience from three pediatric institutions with review of the literature. Brain Pathol Zurich Switz 31:70-83

8. Chadda KR, Holland K, Scoffings D, Dean A, Pickles JC, Behjati S, et al. (2021) A rare case of paediatric astroblastoma with concomitant MN1GTSE1 and EWSR1-PATZ1 gene fusions altering management. Neuropathol Appl Neurobiol
9. Qaddoumi I, Orisme W, Wen J, Santiago T, Gupta K, Dalton JD et al (2016) Genetic alterations in uncommon low-grade neuroepithelial tumors: BRAF, FGFR1, and MYB mutations occur at high frequency and align with morphology. Acta Neuropathol (Berl) 131:833-845

10. Johnson A, Severson E, Gay L, Vergilio J, Elvin J, Suh J et al (2017) Comprehensive genomic profiling of 282 pediatric low- and high-grade gliomas reveals genomic drivers, tumor mutational burden, and hypermutation signatures. Oncologist 22:1478-1490

11. Burel-Vandenbos F, Pierron G, Thomas C, Reynaud S, Gregoire V, Duhil de Benaze G, et al. (2020) A polyphenotypic malignant paediatric brain tumour presenting a MN1-PATZ1 fusion, no epigenetic similarities with CNS High-Grade Neuroepithelial Tumour with MN1 Alteration (CNS HGNET-MN1) and related to PATZ1-fused sarcomas. Neuropathol Appl Neurobiol, 46: 506-509

12. Louis DN, Perry A, Wesseling P, Brat DJ, Cree IA, Figarella-Branger D, et al. (2021) The 2021 WHO classification of tumors of the central nervous system: a summary. Neuro-Oncol., noab106.

13. Capper D, Jones DTW, Sill M, Hovestadt V, Schrimpf D, Sturm D et al (2018) DNA methylation-based classification of central nervous system tumours. Nature 555:469-474

14. Tauziède-Espariat A, Pagès $M$, Roux A, Siegfried A, Uro-Coste E, Nicaise Y et al (2019) Pediatric methylation class HGNET-MN1: unresolved issues with terminology and grading. Acta Neuropathol Commun 7:176

15. Park KW, Cai Y, Benjamin T, Qorbani A, George J (2020) Round cell sarcoma with EWSR1-PATZ1 gene fusion in the neck: case report and review of the literature. Laryngoscope 130:E833-E836

16. Chougule A, Taylor M, Nardi V, Chebib I, Cote GM, Choy E et al (2019) Spindle and round cell sarcoma with EWSR1-PATZ1 gene fusion: a sarcoma with polyphenotypic differentiation. Am J Surg Pathol 43:220-228

17. Michal M, Rubin BP, Agaimy A, Kosemehmetoglu K, Rudzinski ER, Linos K, et al. (2021) EWSR1-PATZ1-rearranged sarcoma: a report of nine cases of spindle and round cell neoplasms with predilection for thoracoabdominal soft tissues and frequent expression of neural and skeletal muscle markers. Mod Pathol Off J U S Can Acad Pathol Inc., 34:770-785

18. Kuo Y-J, Lewis JS, Zhai C, Chen Y-A, Chernock RD, Hsieh M-S, et al. (2021) DEK-AFF2 fusion-associated papillary squamous cell carcinoma of the sinonasal tract: clinicopathologic characterization of seven cases with deceptively bland morphology. Mod Pathol Off J U S Can Acad Pathol Inc., 34: 1820-1830

19. Rooper LM, Agaimy A, Dickson BC, Dueber JC, Eberhart CG, Gagan J, et al. (2021) DEK-AFF2 carcinoma of the sinonasal region and skull base: detailed clinicopathologic characterization of a distinctive entity. Am J Surg Pathol.

20. Li B, Qu H, Zhang J, Pan W, Liu M, Yan X et al (2021) Genomic characterization and outcome evaluation of kinome fusions in lung cancer revealed novel druggable fusions. NPJ Precis Oncol 5:81

21. Ohki K, Kiyokawa N, Watanabe S, Iwafuchi H, Nakazawa A, Ishiwata K et al (2021) Characteristics of genetic alterations of peripheral T-cell lymphoma in childhood including identification of novel fusion genes: the Japan Children's Cancer Group (JCCG). Br J Haematol 194:718-729

22. Bensaid M, Melko M, Bechara EG, Davidovic L, Berretta A, Catania MV et al (2009) FRAXE-associated mental retardation protein (FMR2) is an RNAbinding protein with high affinity for G-quartet RNA forming structure. Nucleic Acids Res 37:1269-1279

23. Hovestadt V, Jones DTW, Picelli S, Wang W, Kool M, Northcott PA et al (2014) Decoding the regulatory landscape of medulloblastoma using DNA methylation sequencing. Nature 510:537-541

\section{Publisher's Note}

Springer Nature remains neutral with regard to jurisdictional claims in published maps and institutional affiliations. 